\title{
Delayed allograft rejection by the suppression of class II transactivator
}

\author{
Tae Woon $\mathrm{Kim}^{1 *}$, Young Mi Choi ${ }^{1 *}$, \\ Jae Nam Seo ${ }^{1}$, Ju Hyun Kim, \\ Young Ho Suh ${ }^{2}$, Doo Hyun Chung ${ }^{3,4,5}$, \\ Kyeong Cheon Jung ${ }^{3,4,6}$ and Kwon Ik Oh ${ }^{1,6}$ \\ ${ }^{1}$ Department of Pathology \\ Hallym University College of Medicine \\ Chuncheon 200-702, Korea \\ ${ }^{2}$ Division of Metabolic Diseases \\ Department of Biomedical Sciences \\ National Institute of Health \\ Seoul 122-701, Korea \\ ${ }^{3}$ Graduate Program of Immunology \\ ${ }^{4}$ Department of Pathology and \\ ${ }^{5}$ Xenotransplantation Research Center \\ Seoul National University College of Medicine \\ Seoul 110-799, Korea \\ ${ }^{6}$ Corresponding authors: Tel, 82-2-740-8261 (KC Jung), \\ 82-33-248-2564 (KI Oh); \\ Fax, 82-2-763-6625 (KC Jung), 82-33-241-8250 (KI Oh); \\ E-mail, jungkc66@snu.ac.kr (KC Jung), \\ kwonik@hallym.ac.kr (KI Oh) \\ *These authors contributed equally to this work.
}

\section{Accepted 6 April 2006}

Abbreviations: CIITA, class II transactivator; DN, dominant-negative; FasL, Fas ligand; KO, knockout

\begin{abstract}
We examined the effect of class II transactivator (CIITA) down-modulation on allograft rejection. To inhibit the function of CIITA, we constructed a series of CIITA mutants and found one exhibiting the dominant-negative effect on the regulation of major histocompatibility complex (MHC) class II expression. To test whether the CIITA dominantnegative mutant reduces immunogenecity, CIITAtransfected melanoma cells were injected into allogeneic host and assessed for immune evading activity against host immune cells. We demonstrated that the CIITA dominant-negative mutant allowed tumor nodules to develop earlier in the lung than control by this tumor challenge study. Furthermore, skin grafts deficient for CIITA also survived longer than wild-type in allogeneic hosts. Both the tumor
\end{abstract}

challenge and skin graft studies suggest the inhibition of CIITA molecules in donor tissue would be beneficial to the control of allo-response.

Keywords: graft rejection; melanoma, experimental; MHC class II transactivator protein; neoplasm transplantation; transplantation, homologous

\section{Introduction}

The complex immune mechanisms that lead to organ-graft rejection originate with the recognition of allogeneic major histocompatibility complex (MHC) antigen complexes by $T$ cells (Watschinger, 1995; Hernandez-Fuentes et al., 1999). Earlier reports showed evidences that MHC plays a significant role in the events leading to the rejection or acceptance of allografts (Bach and Sachs, 1987; Krensky and Clayberger, 1993; Shoskes and Wood, 1994). Likewise, MHC disparate skin or heart were rejected rapidly in CD8 deficient recipients but were accepted in CD4 deficient recipients (Krieger et al., 1996; Pietra et al., 2000), demonstrating the critical interaction between MHC class II and CD4 T cells for immune destruction of grafted tissues.

MHC class II molecules are constitutively expressed only on few cell types such as B cells, thymic epithelial cells and mature dendritic cells. However, class II expression can be induced in many cell types by a variety of stimuli, such as IFN- $\gamma$ (Boss, 1997). A non-DNA binding protein, class II transactivator (CIITA), has been identified as the pivotal master regulator that controls tissue specific and inducible control of class II and antigen processing gene expression (Steimle et al., 1993; Ting and Trowsdale, 2002). In addition, CIITA up-regulates the expression of MHC class I through a similar set of promoter elements (Martin et al., 1997). Recently, the generation of CIITA knockout (CIITA KO) mice (Chang et al., 1996) has confirmed the requirement of CIITA for regulated expression of $\mathrm{MHC}$ class II. This renders CIITA an ideal target for enhancing graft survival.

In this study, we investigated the role of CIITA in transplantation rejection using allogeneic tumor cells expressing CIITA dominant-negative (CIITA DN) and skin tissue of CIITA KO mice. Down-modulation of CIITA allows the tumor cells to grow more rapidly. In addition, CIITA deficient allografts showed enhanc- 
ed survival compared to the wild-type control.

\section{Materials and Methods}

\section{Animals and Cell lines}

Ten- to 12-week-old male C57BL/6 $\left(\mathrm{H}-2^{\mathrm{b}}\right)$ and BALB/C $\left(\mathrm{H}-2^{\mathrm{d}}\right)$ mice were purchased from Daehan Biolink (Chunduk, Korea). Mice were housed in a conventional facility at Hallym University (Chuncheon, Korea). All experimental animals were cared for, maintained, and terminated in accordance with the Hallym University Guideline. The B16-F1 murine melanoma cell line (obtained from American Type Culture Collection, Manassas, VA) was maintained in DMEM supplemented with 10\% FCS, $2 \mathrm{mM}$ L-glutamine and $0.1 \%$ penicillin/streptomycin (GIBCOBRL, Grand Island, NY).

\section{Retroviral transduction}

The diagrammatic representation of the human CIITA cDNA and its mutants is given in Figure 1. They were cloned into pRetro-vector. Retroviral packaging and transduction were done as previously described (Ory et al., 1996). Briefly, plasmid DNA were transfected into the B16 cell line using calcium phosphate precipitation and forty-eight hours after transfection, collected the supernatant, sterilized by filtration and stored for later use at $-70^{\circ} \mathrm{C}$. Cells were transduced by adding $0.5 \mathrm{ml}$ of viral supernatant with $4 \mu \mathrm{g} / \mathrm{ml}$ of polybrene (Sigma, St. Louise, MO) for $2 \mathrm{~h}$ and single clones expressing GFP highly were selected using limiting dilution assay.

\section{IFN- $\gamma$ treatment}

B16 cells $\left(2 \times 10^{5}\right)$ were cultured for $24 \mathrm{~h}$ and then incubated with mouse IFN- $\gamma(500 \mathrm{U} / \mathrm{ml}$; Boehringer Mannheim, Indianapolis, IN) for $72 \mathrm{~h}$. B16 cells were stained with appropriate antibodies or used for other functional assay.

\section{Flow cytometry analysis}

For flow cytometry, cells in log growth phase were harvested and washed twice with $1 \times$ PBS containing $0.05 \%$ sodium azide (Zhang et al., 2005). The cells $\left(1 \times 10^{6}\right)$ were incubated for 30 min with diluted primary antibodies, washed three times and then analyzed immediately. Phycoerythrin (PE)-conjugated anti-mouse $\mathrm{MHC}$ class I and II were
A

Full CIITA cDNA

\begin{tabular}{|c|c|c|}
\hline $\begin{array}{c}A-D \\
1-125\end{array}$ & $\begin{array}{c}\text { PST-D } \\
126-322\end{array}$ & $\begin{array}{c}\text { GTP-D } \\
336-702\end{array}$ \\
\hline
\end{tabular}

B Dominant negative CIITA
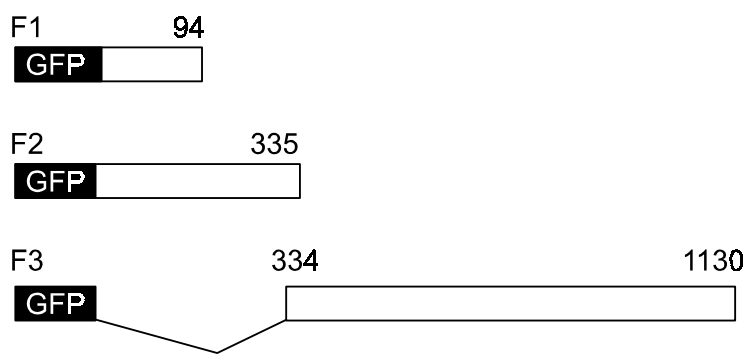

C

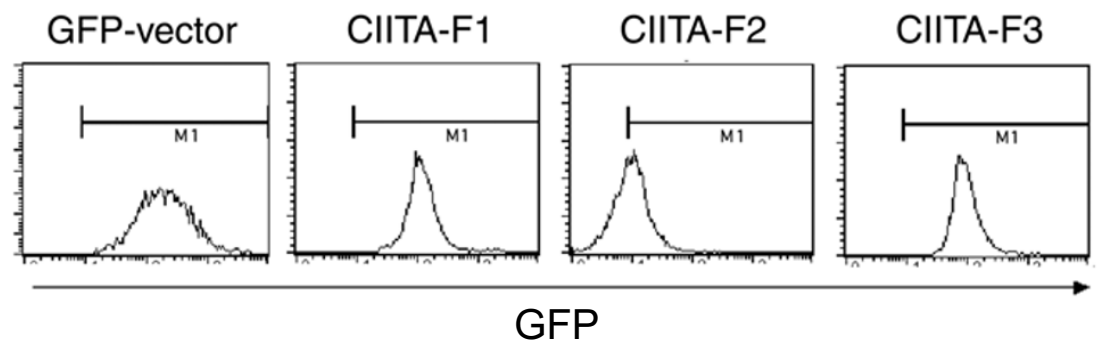

Figure 1. The structures of wild-type CIITA and dominant-negative effector proteins (F1, F2 and F3). The different CIITA constructs are schematically represented. Position of the acidic, PST and GTP binding domains are indicated (A). Numbers indicate that the N- and C-terminal amino-acid positions of the mutant CIITA constructs (B). Each gene constructs were introduced into melanoma cell line and the clones expressing GFP selected for the further study $(\mathrm{C})$. 
A

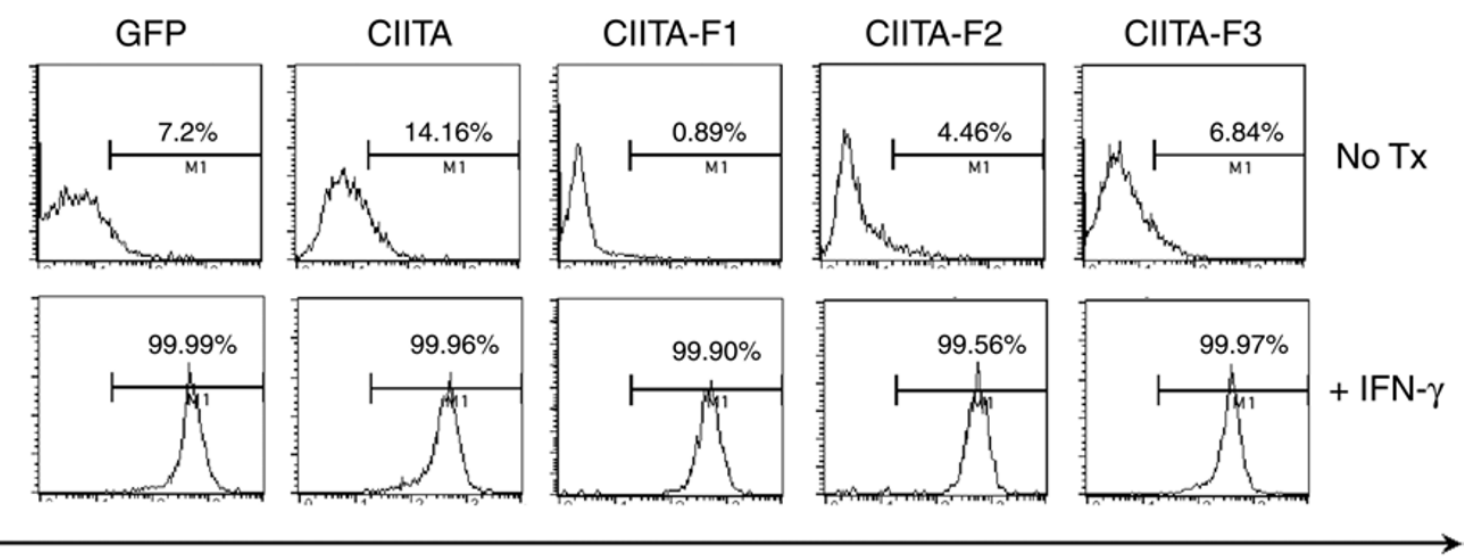

MHC class I

B

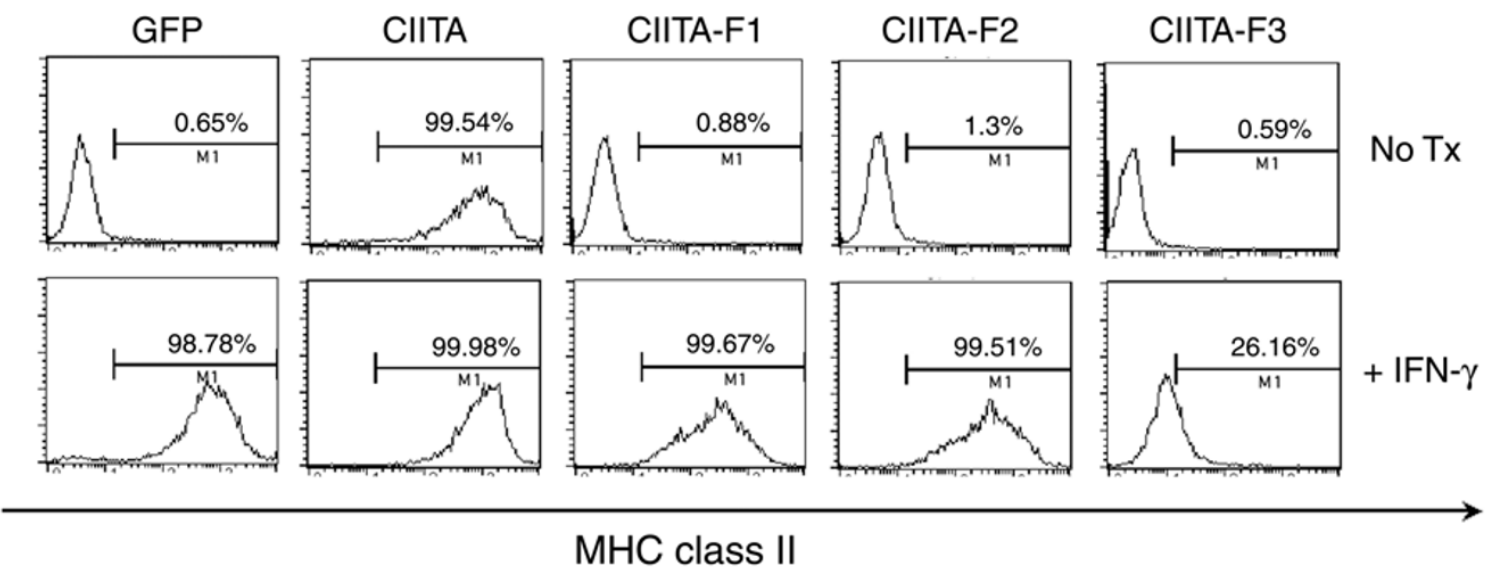

Figure 2. Repression of IFN- $\gamma$ induced MHC class II expression by dominant negative CIITA-F3 mutant. Flow cytometry analysis of MHC class II (A) and I (B) in stably transfected B16 cells is shown. B16 cells in lower panels of (A) and (B) were treated with mouse IFN- $\gamma$ at the concentration of 500 $\mathrm{U} / \mathrm{ml}$ in the culture medium for $72 \mathrm{~h}$ while those in upper panels were not.

purchased from BD Pharmingen (San Diego, CA). The flow cytometric analysis was performed using a FACSCalibur (BD BioSciences, Mountain View, CA).

\section{Injection of mice and identification of the tumor nodules}

B16 cells ( $>95 \%$ viable by trypan blue exclusion assay) were suspended at a concentration of $1 \times$ $10^{6} / \mathrm{ml}$ in PBS, and then injected intravenously into a lateral tail vein of C57BL/6 or BALB/c mice $(200$ $\mu \mathrm{l} /$ mouse). Three-to-four weeks later, mice were euthanized, and the lung was removed and examined macroscopically for the development of tumor nodules (Kim et al., 2004; Chang et al., 2005).

\section{Skin grafting}

BALB/C ( $H-2^{d}$ background) mice were challenged with a full-thickness tail skin graft from either wildtype $\mathrm{B} 6$ or CIITA KO $\left(\mathrm{H}-2^{b}\right)$ mice. Tail skin graft were examined daily and scored on a scale from 1 to 4 for redness, dryness, scaliness and the presence and quality of hair. Grafts were considered rejected when any one of the above characteristics received a score of 4 .

\section{Statistical analysis}

An unpaired Student's $t$ test was used to examine differences in the number of metastatic nodules in the lung and the survival time of skin allografts. This statistical analysis was determined using GraphPad Prism (GraphPad Software, Inc., San Diego, CA). 
A

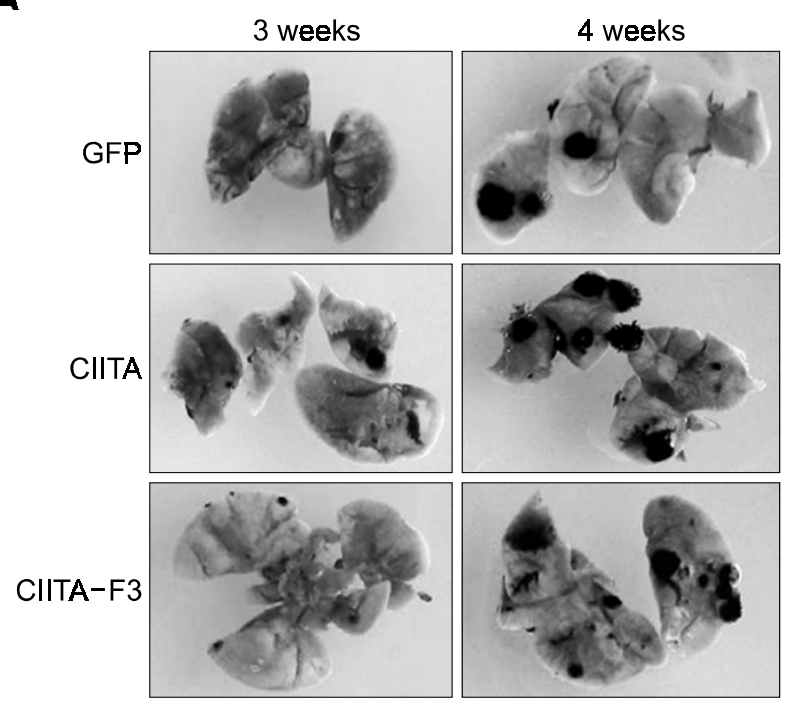

B

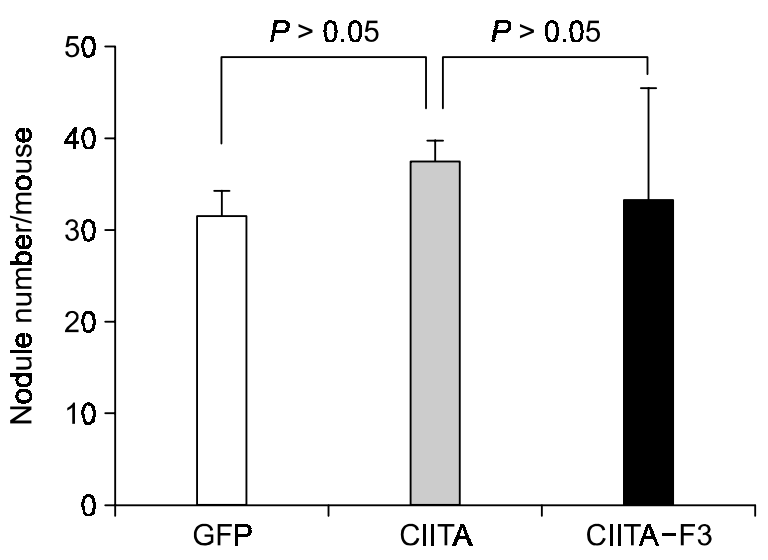

Figure 3. The rates of tumor growth in syngeneic host are not significantly different among various B16 clones irrespective of CIITA or MHC class II expression. Each B16 clone expressing three different CIITA molecules were injected into the tail vein of syngeneic B6 mice at $2 \times 10^{5}$ cells $/$ mouse. Three-to-four weeks later, mice were sacrificed and the lungs were examined to identify tumor nodules.

\section{Results}

IFN- $\gamma$ fails to induce the expression of MHC II molecules in B16 cells expressing CIITA-F3 mutant

The $\mathrm{N}$ terminus of CIITA contains an acidic domain, followed by PST domain rich in proline (amino acids 163-195), serine (amino acids 209-237), and threonine (amino acids 260-322) (Boss, 1997; Sisk et al., 2001; Ting and Trowsdale, 2002) and the previous report showed these acidic or PST domains are important in the regulation of MHC class II expression (Chin et al., 1997). Therefore, to find the CIITA mutant to exhibit dominant-negative properties on MHC class II expression, three different gene constructs in which either the acidic or PST domains were deleted were cloned (CIITA-F1, 2 and 3, Figure 1A). In addition to the CIITA mutant, wild-type CIITA proteins were fused in-frame to the $\mathrm{C}$-terminus of GFP, and termed CIITA (wild-type). Each gene construct was introduced into B16 murine melanoma cell line using retroviral transduction and the clones intensely expressing GFP (as a marker for each CIITA mutant protein) were selected and used in the further experiments (Figure 1C).

The administration of IFN- $\gamma$ activates the Jak/Stat signaling cascade and CIITA transcription in many cells and cell lines (Steimle et al., 1994; Miller et al., 1998). This induction is critical for the function of antigen-presenting cells. To identify which type of CIITA mutants block the above effect, CIITA mutants were treated with $500 \mathrm{U} / \mathrm{ml}$ of IFN- $\gamma$ for 3 days, stained with the antibody against I-A/I-E and then analyzed by FACS. In GFP, CIITA, CIITA-F1 and -F2 clones MHC class II molecules were highly induced by IFN- $\gamma$ stimulation. However, in $\mathrm{F} 3$ clones the induction of $\mathrm{MHC}$ class II was significantly blocked even in the presence of IFN- $\gamma$ (Figure 2A). This inhibitory effect was specific, since the expression levels of MHC class I were not affected (Figure 2B). These results clearly demonstrate that CIITA-F3 has dominant-negative property in $\mathrm{MHC}$ class II expression.

\section{F3 mutant suppresses allogeneic rejection}

Using the B16 cell lines (GFP control, CIITA, CIITAF3) as mentioned above, we investigated the possible roles of CIITA in tumor immunogenicity that could be mediated by MHC class I, MHC class II, or both. To test whether CIITA-F3 cells affect tumor immunity, we immunized syngeneic B6 or allogeneic BALB/c mice with B16 cells. In consistent with the previous report (Martin et al., 1999), we could not detect any difference in the rate or extent of the development of tumor nodules of the lung among three different B16 cell lines in syngeneic B6 mice (Figure $3, P>0.05$ ). However, the suppression of CIITA function renders tumor nodules to develop earlier in allogeneic hosts. While we could not detect the metastatic nodules in the mice immunized with GFP or other CIITA transfectants, several metastatic nodules were identified in those immunized with F3 mutants in 3-4 weeks and the numbers or sizes of those nodules were almost the same as those observed in B6 mice (Figure 4, $P<0.05$ ). 
A

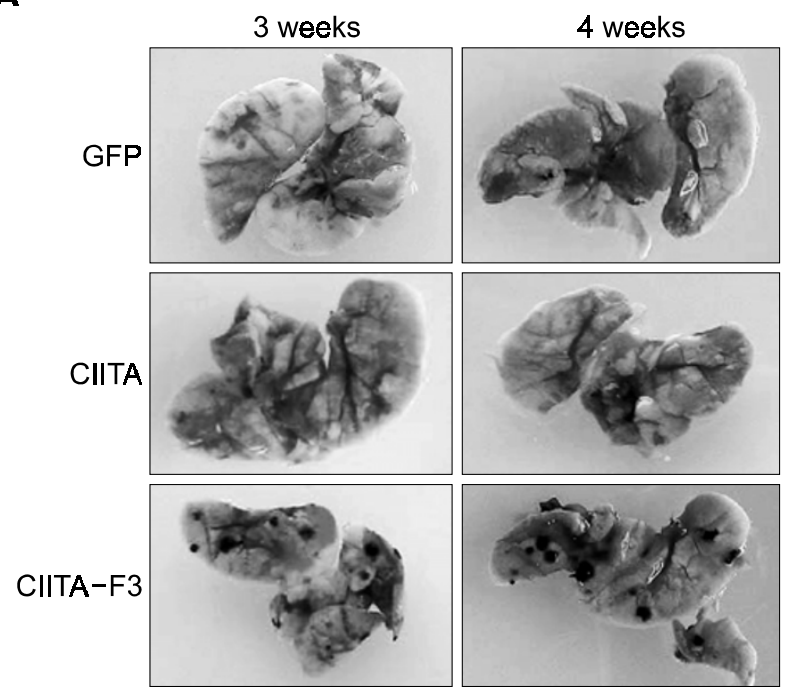

B

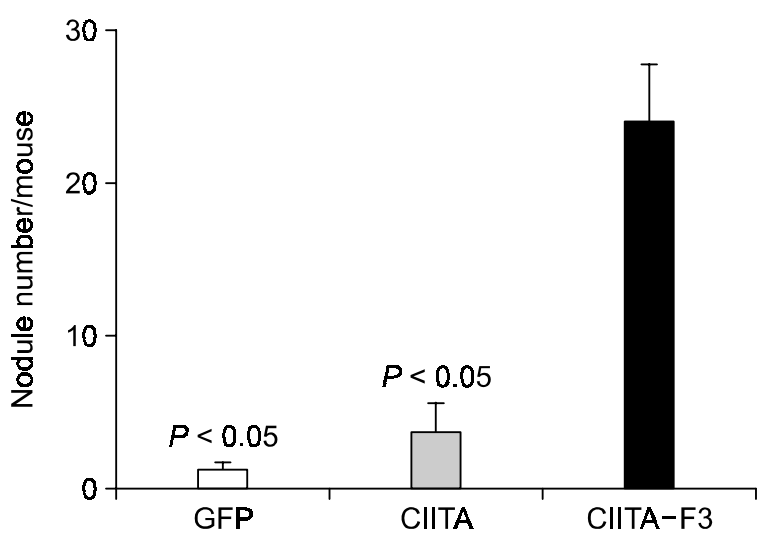

Figure 4. Increased number of tumor nodules in B16 cells expressing CIITA-F3 muatnt in allogeneic host. (A) Three different B16 cells were administrated into allogeneic BALB/c mice and the tumor nodules were examined as described in Figure 3. (B) Statistical analysis of the number of tumor nodules derived from B16 cells expressing CIITA variants was done using Prism software (GraphPad Software, Inc., San Diego, CA).

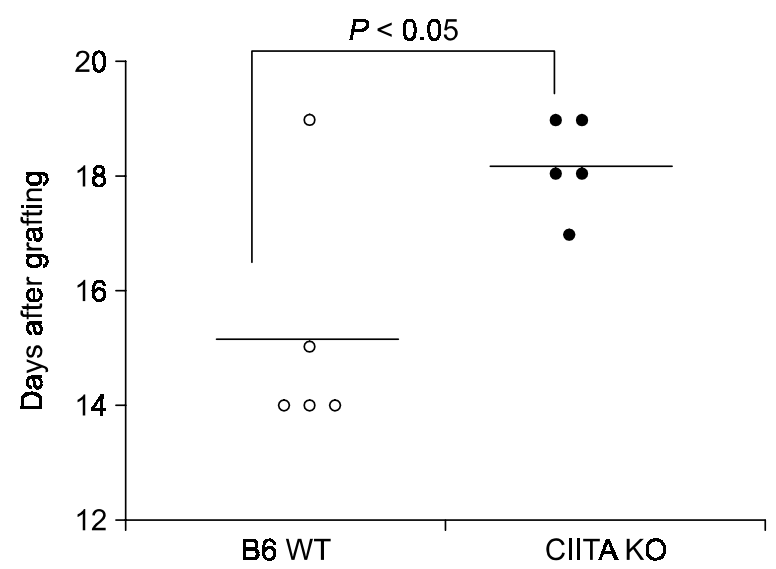

Figure 5. Survival of skin allografts from B6-wild type (B6-WT, $\mathrm{H}-2^{\mathrm{b}}$ haplotype) and $\mathrm{CIITA} \mathrm{KO}\left(\mathrm{H}-2^{\mathrm{b}}\right)$ on allgeneic recipients. Allogeneic $B A L B / C\left(H-2^{d}\right)$ recipients mice were challenged with tail skin grafts from B6-WT or CIITA KO mice. CIITA KO allografts, in contrast to control B6-WT, have a delayed rejection rate $(P<0.05)$. Statistical significance was determined by unpaired $t$ test.

\section{Loss of CIITA function prolongs graft survival}

The above data suggest that the down-modulation of CIITA increases the survival of allograft. We address this issue using skin graft model. When skin tissues from either wild-type $\mathrm{B} 6$ or CIITA $\mathrm{KO}\left(\mathrm{H}-2^{\mathrm{b}}\right.$ background) mice were grafted into allogeneic BALB/c $\left(\mathrm{H}-2^{\mathrm{d}}\right)$ recipients, we anticipated the production of vigorous allogeneic immune responses caused by the incompatibility of major and minor histocompatibility antigens between donor and recipient.
However, CIITA KO skin grafts survived significantly longer, with a mean survival time of $18 \pm 0.38$ days (mean \pm SEM), even though wild-type mismatched allografts were rejected rapidly within $15.25 \pm 0.59$ days after transplantation (Figure $5, P<0.05$ ). Altogether, these results demonstrate that the down-modulation of CIITA can reduce the rate of allorejection in vivo.

\section{Discussion}

The consequences of inhibition of CIITA function in allo-response were examined using two models: tumor challenge and skin graft experiments. Although we could not detect any evidence that the modulation of CIITA in donor cells induces a change of immune response in syngeneic recipients, inhibition of CIITA function allowed recipients more tolerable to the grafted tissues in allogeneic recipients. In skin graft experiment, the skin from CIITA $\mathrm{KO}$ mice survives significantly longer than that from B6 wild-type mice. In consistent with the results, the tumor growth in BALB/c mice immunized with B16 cells containing CIITA dominant-negative was slower and less aggressive, compared with control GFP. However, even though the above data show that CIITA is necessary for the development of acute rejection, the inhibitory effect of CIITA mutants is not so complete for donor to accept the graft permanently, but just to delay the response. Therefore, other proteins except CIITA are considered to play a major role in allograft rejection. 
There are several plausible reasons why the differences between wild-type and F3/CIITA KO are not either extensive or permanent. First of all, although direct reactivity of recipient $T$ cells with donor antigen presenting cells is considered to play a role in allograft rejection, indirect presentation of donor antigen by recipient APC to recipient $\mathrm{T}$ cells needs to be considered. MHC-derived peptides, when presented indirectly by way of recipient $\mathrm{MHC}$ in vitro, produce the most vigorous responses (Gould and Auchincloss, 1999). In addition, although CIITA serves as a master-regulator of MHC class II molecule, CIITA-independent controlled mechanisms could allow the production of MHC class II (Boss, 1997). Thus, these proteins in CIITA deficient allografts could serve as antigenic peptides for indirect presentation by host APC to host T cells or could be inappropriately presented by class I molecules.

Although CIITA functions primarily to regulate $\mathrm{MHC}$ class II gene expression, it may also regulate the expression of other genes. Among many reports, of greater significance is the recent finding that CIITA can inhibit Fas ligand (FasL) expression, presumably through interference with nuclear factor of activated T cells (Zhu and Ting, 2001; Gourley and Chang, 2001). In CIITA KO allografts or B16 cell containing CIITA-F3 mutants, absence of CIITA might lead to increased expression of FasL, which in turn might favor activation-induced cell death. This could facilitate the development of allograft tolerance by promoting the deletion of effector T cells.

The growing base of knowledge about the mechanisms of CIITA action suggests that molecular and therapeutic tools for inhibiting CIITA function could have great utility. In this report, we show that down-modulation of CIITA can be used for suppression of allo-geneic immune response. Additional fine mapping and mutagenesis studies could lead to the design of peptides that could specifically prevent CIITA action in situ and have a therapeutic potential for allo-rejection.

\section{Acknowledgement}

This study was supported by a National R\&D Program Grant of The Ministry of Science and Technology (M1031006005-03B4606-00510).

\section{References}

Bach FH, Sachs DH. Current concepts: immunology. Transplantation immunology. N Engl J Med 1987;317:489-92

Boss JM. Regulation of transcription of MHC class II genes. Curr Opin Immunol 1997;9:107-13

Chang $\mathrm{CH}$, Guerder S, Hong SC, van Ewijk W, Flavell RA. Mice lacking the MHC class II transactivator (CIITA) show tis- sue-specific impairment of MHC class II expression. Immunity 1996;4:167-78

Chang M, Lee W, Choi J, Park S, Paik S, Kim YS. Antitumor immunity induced by tumor cells engineered to express a membrane-bound form of IL-2. Exp Mol Med 2005;37:240-9

Chin KC, Li GG, Ting JP. Importance of acidic, proline/serine/threonine-rich, and GTP-binding regions in the major histocompatibility complex class II transactivator: generation of transdominant-negative mutants. Proc Natl Acad Sci USA 1997;94:2501-6

Gould DS, Auchincloss H Jr. Direct and indirect recognition: the role of $\mathrm{MHC}$ antigens in graft rejection. Immunol Today 1999;20:77-82

Gourley TS, Chang $\mathrm{CH}$. Cutting edge: the class II transactivator prevents activation-induced cell death by inhibiting Fas ligand gene expression. J Immunol 2001;166:2917-21

Hernandez-Fuentes MP, Baker RJ, Lechler RI. The alloresponse. Rev Immunogenet 1999;1:282-96

Kim A, Noh Y, Kim KD, Jang Y, Choe Y, Lim J. Activated natural killer cell-mediated immunity is required for the inhibition of tumor metastasis by dendritic cell vaccination. Exp Mol Med 2004;36:428-43

Krensky AM, Clayberger $\mathrm{C}$. The nature of allorecognition. Curr Opin Nephrol Hypertens 1993;2:898-903

Krieger NR, Yin DP, Fathman CG. CD4+ but not CD8+ cells are essential for allorejection. J Exp Med 1996;184:2013-8

Martin BK, Chin KC, Olsen JC, Skinner CA, Dey A, Ozato K, Ting JP. Induction of MHC class I expression by the MHC class II transactivator CIITA. Immunity 1997;6:591-600

Martin BK, Frelinger JG, Ting JP. Combination gene therapy with CD86 and the MHC class II transactivator in the control of lung tumor growth. J Immunol 1999;162:6663-70

Miller DM, Rahill B M, Boss JM, Lairmore MD, Durbin JE, Waldman JW, Sedmak DD. Human cytomegalovirus inhibits major histocompatibility complex class II expression by disruption of the Jak/Stat pathway. J Exp Med 1998;187:675-83

Ory DS, Neugeboren BA, Mulligan RC. A stable human-derived packaging cell line for production of high titer retrovirus/vesicular stomatitis virus $G$ pseudotypes. Proc Natl Acad Sci USA 1996;93:11400-6

Pietra BA, Wiseman A, Bolwerk A, Rizeq M, Gill RG. CD4 T cell-mediated cardiac allograft rejection requires donor but not host MHC class II. J Clin Invest 2000;106:1003-10

Shoskes DA, Wood KJ. Indirect presentation of MHC antigens in transplantation. Immunol Today 1994;15:32-8

Sisk TJ, Roys S, Chang CH. Self-association of CIITA and its transactivation potential. Mol Cell Biol 2001;21:4919-28

Steimle V, Otten LA, Zufferey M, Mach B. Complementation cloning of an $\mathrm{MHC}$ class II transactivator mutated in hereditary $\mathrm{MHC}$ class II deficiency (or bare lymphocyte syndrome). Cell 1993;75:135-46

Steimle V, Siegrist CA, Mottet A, Lisowska-Grospierre B, Mach $B$. Regulation of MHC class II expression by interferon-gamma mediated by the transactivator gene CIITA. Science 1994; 


\section{5:106-9}

Ting JP, Trowsdale J. Genetic control of MHC class II expression. Cell 2002;109 Suppl:S21-33

Watschinger $B$. How $T$ cells recognize alloantigen: evidence for two pathways of allorecognition. Nephrol Dial Transplant 1995;10:1556-8

Zhang S, Park K, Oh S, Cho H, Cho H, Park J, Cho Y, Koo B,
Chae I, Choi D, Kim H, Lee M. NF-кB decoy potentiates the effects of radiation on vascular smooth muscle cells by enhancing apoptosis. Exp Mol Med 2005;37:18-26

Zhu XS, Ting JP. A 36-amino-acid region of CIITA is an effective inhibitor of CBP: novel mechanism of gamma interferon-mediated suppression of collagen alpha(2)(I) and other promoters. Mol Cell Biol 2001;21:7078-88 